\title{
Ultrasonographically determined renal values and comparisons to serum biochemistry renal variables in aged semi-captive cheetahs (Acinonyx jubatus)
}

\author{
Robert M. Kirberger ${ }^{1 *}$ (D) and Adrian S. W. Tordiffe $e^{2,3}$
}

\begin{abstract}
Background: Cheetahs in captivity have a high prevalence of chronic renal diseases. We ultrasonographically evaluated the renal volumes, a variety of renal dimensions, interarcuate artery resistive indices (RI) as well as aortic diameters and the length of the ventral aspect of the 6th lumbar vertebrae in 27 aged semi-captive anesthetized cheetahs. Renal size, dimensions and ratios were compared to urine specific gravity, serum creatinine and urea values.

Results: There were minimal differences for all values between left and right kidneys. Mean kidney length was $65.1 \mathrm{~mm}$ (range 55.2-76.9) with left kidney length ratios to L6 length being 1.60 (range 1.27-2.06) and to the aortic diameter 7.69 (range 4.54-10.72). Significant correlations between left renal length as well as length:L6 ratio to creatinine values were found $((r-0.66)$ and $(r-0.60)$ respectively). The mean RI values of the different sedation/anesthetic protocols ranged from $0.46-0.55$.

Conclusions: Left renal length and L6 ventral vertebral body length as well as left kidney RI values should be routinely measured in all cheetah abdominal ultrasound examinations. These measurements, together with serum creatinine, urea and urine specific gravity values may be relatively sensitive indicators of early renal pathology in the absence of gross ultrasonographic changes.
\end{abstract}

Keywords: Cheetah, Kidney function, Measurements, Diagnostic ultrasound

\section{Background}

The normal ultrasonographic anatomy of the cheetah liver, kidneys, urinary tract [1], adrenals [2] and female reproductive system have been described [3]. Diagnostic ultrasonography has been shown to be a valuable diagnostic tool, particularly under field conditions, to evaluate normal ultrasonographic abdominal anatomy as well as reproductive cyclical changes and has the potential to diagnose pathology as is routinely performed in domestic animals.

Chronic renal disease is known to be a common cause of morbidity and mortality in captive cheetahs. Nephropathies recorded at post-mortem include glomerulonephritis,

\footnotetext{
* Correspondence: Robert.kirberger@up.ac.za

${ }^{1}$ Department of Companion Animal Clinical Studies, Faculty of Veterinary Science, University of Pretoria, Private Bag X04, Onderstepoort 0110, South Africa

Full list of author information is available at the end of the article
}

glomerulosclerosis, renal fibrosis, acute tubular necrosis, interstitial nephritis, oxalate nephrosis, pyelonephritis and renal amyloidosis [4-10]. Of these, glomerulosclerosis and renal fibrosis tend to be the most common lesions, typically present in $70-80 \%$ of renal samples evaluated on histology in adult captive cheetahs $[4,5,9]$.

Renal function can be evaluated by a variety of laboratory procedures including the determination of blood urea nitrogen (BUN) and creatinine concentrations. Urine protein to creatinine ratios as well as electrolyte fractional excretion ratios can also be determined. The serum concentration of symmetric dimethylarginine (SDMA) has also been shown to be a more useful indicator of early renal disease in domestic dogs and cats $[11,12]$, but its use has not yet been formally evaluated in cheetahs. Urine specific gravity and sediment evaluation are also potential indicators of renal disease. Some 
serum biochemical analytes have been reported specifically for cheetahs $[13,14]$. A maximum serum creatinine concentration of $300 \mu \mathrm{mol} / \mathrm{L}$ has been suggested to reflect normal renal function in cheetahs [15] and this is supported by a more recent study in 66 apparently healthy cheetahs where a maximum concentration of $303 \mu \mathrm{mol} / \mathrm{L}$ was recorded [14].

A number of renal size imaging measurement techniques have been made in veterinary practice which could provide clinically useful information regarding possible underlying renal disease. These include computed tomography and radiography, which are usually not readily available under field conditions, and diagnostic ultrasound. The latter is readily accessible, has no ionizing radiation, does not require the use of intravenous iodine preparations to enhance renal visibility and can be performed repeatedly [16]. Ultrasound determined renal volumes in man have been shown to have a weak positive correlation with various glomerular filtration rate indices and could be a valuable prognosticator for the severity of chronic renal disease in resource poor human communities [17]. Similar findings could be applicable to cheetahs where sophisticated medical equipment is not always readily accessible. Additionally, serial volume determinations may be more accurate than single linear measurements to detect changes in renal size [18].

Radiographic renal size has been reported in normal captive cheetahs [19]. The ratio of the length of each kidney to the length of the second lumbar vertebral body (L2) was determined. There was no significant difference in this ratio between left and right kidneys with values ranging from 1.53-2.09. This was smaller than the ratio reported in ventrodorsal abdominal radiographs of domestic cats, which ranged from 1.9-2.6 times the length of L2 in neutered cats to 2.1-3.2 in entire cats [20].

Ultrasonographically determined renal size has been reported in dogs [21, 22], cats [23] as well as in the cheetah [1]. This may simplistically be done as renal length, width or height, or more accurately by determining renal volume. Volume measurements may be a more reliable indication of renal size than renal length. $\mathrm{Nu}$ merous techniques have been described in dogs [18, 24] and cats [25]. Volumes can be determined using prolate ellipsoid geometric methods from linear and crosssectional area measurements. Compared to the gold standard volume displacement method, the single plane area-length method had the best correlation $(r=0.94)$ [24]. Alternatively, and easier and faster to do, only linear measurements can be used to determine volume using a variety of similar formulas [18]. In the latter study the best correlation $(r=0.98)$ was obtained compared to the volume displacement method by using the following formula

$$
\mathrm{V}=\mathrm{L} X\left(\frac{\mathrm{W} 1+\mathrm{W} 2}{2}\right) \times\left(\frac{\mathrm{H} 1+\mathrm{H} 2}{2}\right) \times\left(\frac{\pi}{6}\right)
$$

where $\mathrm{V}=$ volume; $\mathrm{L}=$ maximum length $\mathrm{W} 1$ and $\mathrm{H} 1$ = width and height at the cranial pole and W2 and $\mathrm{H} 2$ the same measurements at the caudal pole.

More recently an ultrasonographically determined kidney-to-aorta ratio has been used to estimate renal size in normal dogs [26]. Renal length was compared to the longitudinal aortic maximum luminal diameter adjacent to the kidneys, just caudal to the origin of the left renal artery. Left and right renal lengths and ratios did not differ significantly with a mean \pm standard deviation (SD) ratio of $7.3 \pm 0.9$ and range of 5.5-9.4.

No reference could be found to compare ultrasonographic renal length or volume to ultrasonographically determined lumbar vertebral body length. The value of diagnostic ultrasound in evaluating the vertebra and in the diagnosis of vertebral pathology, has been proven in dogs [27]. The ventral borders of the lumbar vertebrae can readily be identified, more so caudally, and the lumbosacral junction identified due to the angle of the sacral vertebral body to the lumbar vertebra in dogs and cats. Assuming seven lumbar vertebra in the cheetah, a caudal lumbar vertebra can be measured as a size comparison for renal length. The standard vertebra used for radiographic ratios, L2, may be more difficult to measure ultrasonographically due to the adjacent kidneys. The L7 length should not be used as in dogs it has been reported that it may be shorter than the remaining lumbar vertebra [20]. The L6 vertebra should thus be readily identifiable and measurable for comparison of renal length.

The Doppler resistive index (RI) is a non-invasive assessment of renal hemodynamics and has been advocated as a useful clinical parameter to detect real disease. The RI is calculated from the intrarenal arterial Doppler flowvelocity waveform as (peak systolic velocity - end diastolic peak velocity)/peak systolic velocity $[28,29]$. Although initially believed to be a reflection of intrarenal disease, RI is a complex interaction between renal and systemic vascular wall properties and hemodynamics factors and in man it has been shown that there is a significant association between RI and central and peripheral pulse pressure [29].

Normal RI values for awake dogs are $0.63 \pm 0.05$ and $0.62 \pm 0.05$ for the left and right kidneys respectively [30]. In a study in dogs sedated with acepromazine maleate followed by diazepam and ketamine hydrochloride the values fell to $0.44 \pm 0.06$ and $0.45 \pm 0.06$ for left and right kidneys, a significant decrease compared to unsedated dogs [31]. In the latter study there was no significant difference between the left and right kidneys. Increased RI values in dogs have been attributed to total ureteral obstruction [30], acute renal disease [31] and 
Addison's disease [32]. In 10 healthy domestic shorthaired cats sedated with acepromazine followed by intravenous ketamine hydrochloride $(10 \mathrm{mg} / \mathrm{kg})$ there was no significant difference between left and right arcuate artery RIs with a mean \pm SD for the left kidney of $0.56 \pm 0.06$ and right kidney $0.59 \pm 0.06$ [33]. In another study of 50 healthy domestic shorthaired cats a similar anesthetic protocol was used but with the addition of xylazine $(1 \mathrm{mg} / \mathrm{kg})$ [23]. Their RI values were slightly lower at $0.52 \pm 0.05$ for the left kidney and $0.55 \pm 0.05$ for the right kidney. In another study comparing the renal RI in 8 awake versus isoflurane-anaesthetized cats, the awake cats had a RI of $0.55 \pm 0.07$ whilst the cats under anesthesia values were $0.93 \pm 0.03$ [34]. The duration of anesthesia prior to RI measurement was not stated. It was believed that the isoflurane produced increased intrarenal vascular resistance and decreased renal blood flow. In a study in awake Persian cats there was no statistical difference between left and right kidney interlobar RI values with a mean of $0.52 \pm 0.06$ [35]. In a more recent feline study comparing healthy cats and cats with renal disease, none of the cats were sedated or anaesthetized [36]. In the 24 healthy cats of various breeds the RI values differed significantly between left $(0.59 \pm 0.08)$ and right kidneys $(0.54 \pm 0.07)$ but implying no major difference between awake and anaesthetized cats when compared to the feline studies described earlier. However both kidneys showed significant increased RI value in cats with acute kidney injury (left $0.72 \pm 0.08$; right $0.74 \pm 0.08$ ) and chronic renal disease (left $0.73 \pm 0.12$; right $0.72 \pm 0.11$ ). One preliminary cheetah study measured RI values in 5 kidneys of 3 cheetahs, all on different anesthetic protocols, and they had a mean RI of 0.58 with a range of $0.47-0.68$ [1].

The primary objective of this study was to determine a variety of renal variables in aged cheetahs by means of abdominal ultrasonography that could in future assist in detecting renal pathology induced dimension, volume or resistive index changes under field conditions. Additionally we sought correlations between ultrasonographically determined renal variables and serum creatinine and urea as well as urine SG to evaluate the possible benefit of these values.

\section{Methods}

\section{Animals}

The project was part of a long-term health and immunecompetence study of captive cheetahs, Adult cheetahs, aged between 6 and 14 years, were received as orphaned wild cubs by the AfriCat Foundation near Otjiwarongo in Namibia. They were accommodated in fenced bushveld camps, ranging from 3.5 to 25 ha in size (average $11.42 \mathrm{ha}$ ) in small groups of two to six males and/or females that were either contracepted with deslorelin (Supralorin ${ }^{\circ}$, Virbac, Australia) implants or surgically sterilized to comply with Namibian legislative requirements. Their diet consisted of approximately 1.2$1.5 \mathrm{~kg}$ of donkey/horse meat and bones provided with a powdered multivitamin/mineral supplement (Predator supplement; V-Tech, Centurion, South Africa) on 6 days of the week and has been previously published $[2,3]$.

Twenty seven cheetahs were examined during a 5 day observation period in July 2016. Each cheetah was only examined once under general anesthesia. Their data (mean and range of age, weight and measurements) are presented in Table 1. The health examination carried out on each animal included a body condition assessment, dental and oral examination, gastric endoscopy, hematology and serum biochemistry, urinalysis and the abdominal ultrasound evaluation. All the animals included in the study displayed normal behavior prior to immobilization and were judged to be in good health. On gastric endoscopy, none of the cheetahs had macroscopic lesions typically associated with severe lymphoplasmacytic gastritis or any evidence of gastric ulcerations. Cheetahs were fasted for $48 \mathrm{~h}$ prior to anesthesia, but had free access to water. Cheetahs were immobilized with a dart gun using either a combination of tiletamine and zolazapam (Zoletil, Virbac, Halfway House, South Africa) $(0.9-1.4 \mathrm{mg} / \mathrm{kg}$ ) and medetomidine (Domitor, Zoetis, Sandton, South Africa) $(0.03-0.045 \mathrm{mg} / \mathrm{kg})$ or a combination of ketamine (Ketamine hydrochloride, Kyron Laboratories, Johannesburg, South Africa) (3.5$5.7 \mathrm{mg} / \mathrm{kg}$ ) and medetomidine $(0.03-0.45 \mathrm{mg} / \mathrm{kg})$. For maintenance, one of two anesthetic protocols, either isoflurane in oxygen (end-tidal isoflurane concentration $1.1 \pm 0.1 \%$; Forane, Abbot Laboratories, Johannesburg, South Africa) or a propofol infusion $(0.1 \mathrm{mg} / \mathrm{kg} / \mathrm{min}$; Fresenius Propoven 1\%, Fresenius Kabi, Johannesburg, South Africa) with supplemental oxygen were used. Following the procedure, lasting on average between 60 and $90 \mathrm{~min}$, the medetomidine was antagonised with atipamazole (Antisedan, Pfizer, Sandton, South Africa) $(0.08-0.12 \mathrm{mg} / \mathrm{kg})$.

Table 1 Cheetah age, weight, body measurement data (means and ranges)

\begin{tabular}{lllll}
\hline & Males & Females & $P$ value & $\begin{array}{l}\text { Males and } \\
\text { females }\end{array}$ \\
\hline Number & 17 & 10 & 27 \\
Age (years) & $8.9(6-14)$ & $9.9(6-14)$ & 0.47 & 9.3 \\
Weight (kg) & $40.4(32.1-45.7)$ & $35.0(29.5-40.7)$ & $0.003^{*}$ & 38.4 \\
Body length (m) & $0.99(0.93-1.1)$ & $0.94(0.88-1.0)$ & $0.004^{*}$ & 0.98 \\
Shoulder & $0.73(0.68-0.77)$ & $0.71(0.68-0.74)$ & $0.02^{*}$ & 0.72 \\
height (m) & & & & \\
Size index & $0.73(0.63-0.84)$ & $0.67(0.63-0.70)$ & $0.001^{*}$ & 0.70 \\
\hline
\end{tabular}

"Significant differences between males and females 


\section{Body weights and measurements}

All the cheetahs were weighed. Body length (BL) and shoulder height $(\mathrm{SH})$ measurements were obtained with the animal in lateral recumbency using a $1.5 \mathrm{~m}$ flexible plastic measuring tape as published previously [2]. The BL measurement was taken from the occiput at the caudal edge of the skull to the base of the tail (at the fold created between the tail and the body when the tail is lifted to 90 degrees), ensuring that the measuring tape remained in contact with body along its entire length. The SH measurements were taken from the dorsal rim of the scapula to the middle of the metacarpal pad with the shoulder, elbow and carpal joints fully extended. A size index (SI) was calculated from using the formula shoulder height $(\mathrm{m}) \mathrm{X}$ body length $(\mathrm{m})$ similar to techniques described previously [37].

\section{Serum and urine variables}

Blood samples were collected from the jugular vein within 20 min of immobilization using a $20 \mathrm{ml}$ syringe and an 18 gauge needle. Blood was immediately transferred to a plain $6 \mathrm{ml}$ serum tube (Vacutainer, Becton, Dickinson and Co, Sandton, South Africa) and allowed to clot on ice for 40 to $60 \mathrm{~min}$. The tubes were centrifuged at $1500 \mathrm{rpm}$ for $10 \mathrm{~min}$ after which the serum was pipetted off into $2 \mathrm{ml}$ cryovials (Thermo Scientific, Germiston, South Africa) and analyzed immediately. Serum creatinine and urea concentrations were determined using the Vetscan ${ }^{\circ}$ Kidney Profile Plus reagent rotor with an onsite Vetscan VS2 ${ }^{\circ}$ analyser (Abaxis Inc., Union City, USA).

Urine was collected within 20 min of immobilization by aseptic urethral catheterization using a 6 FG dog urinary catheter. Urine was aspirated into a $20 \mathrm{ml}$ syringe and allowed to cool to room temperature before the SG was determined with a calibrated handheld refractometer (RHC-200ATC, Huake Instrument Co., Shenzhen, China).

\section{Ultrasonography}

Each cheetah had its abdomen clipped before undergoing a dorsally recumbent trans-abdominal ultrasound examination of the abdomen. A portable ultrasound machine was used with a 3-6 MHz convex array transducer for the general abdominal examination and a $5-10 \mathrm{MHz}$ linear array transducer set at $10 \mathrm{MHz}$ for evaluation of the gastrointestinal tract and adrenal glands (Mindray Model M7 Vet, Shenzhen Mindray Biomedical from Lomaen, Johannesburg, South Africa) as published previously $[2,3]$.

All measurements were made by one operator (RMK), recorded only once, except for RI, and were reported as mean, range and standard deviation. Each kidney had its length (Fig. 1a) and cranial and caudal pole maximum width and height measured. The length of the ventral aspect of ventral vertebral body of the second last lumbar vertebra (presumed to be L6), was measured (Fig. 1b). This was seen as a concave hyperechoic interface with acoustic shadowing with signal void in the adjacent disc spaces. The aortic luminal diameter was measured just caudal to the left renal artery exit from the longitudinal plane as described previously by Mareschal et al. [26]. The renal volumes were then calculated by means of the prolate ellipsoid technique using the average of the cranial and caudal pole measurements as described by Nyland et al. [18]. Ratios of the renal length and volume to the length of L6, width of the aorta and SI were then determined.

The RI was determined by placing the pulsed wave sampling gate of the $10 \mathrm{MHz}$ linear array transducer in an interlobar artery at the corticomedullary junction region using color flow Doppler. The lowest peak velocity scale was selected that allowed the wave form to be traced without aliasing. Three similar wave forms were measured and the mean RI calculated. As numerous clinical procedures had to be performed on the cheetahs only left renal values were determined to limit anesthetic time. The RI measurements were compared between the two immobilization darting protocols and the two anesthesia maintenance protocols.

Since male cheetahs were significantly larger than females, the renal measurements were also analyzed using values that were corrected for body size, by dividing the various renal values by the SI.

\section{Statistical analyses}

Data were reported as means \pm SDs unless otherwise indicated. All measurements, other than age, serum creatinine and urea were normally distributed as assessed by Shapiro-Wilk's test $(P>0.05)$. Homogeneity of variance was demonstrated in males and females for all measurements, except for SI, using Levene's test for equality of variance. Unpaired t-tests were used to compare the weights, body size, and renal measurements of males and females. Paired-sample t-tests were used to evaluate the differences between left and right renal measurements. A Mann-Whitney $U$ test was run to compare the ages, serum creatinine and urea values of males and females. Spearman's rank correlations were used to evaluate the influence of age, serum urea, serum creatinine and urine SG on the various renal measurements. All analyses were performed with SPSS version 23.0 for Windows (IBM Corporation). Statistical significance was two-tailed and defined as $P<0.05$ in all cases.

\section{Results}

Cheetah weights, body measurements and size index differed significantly between males and females (Table 1 ). Serum and urine variables did not differ significantly 

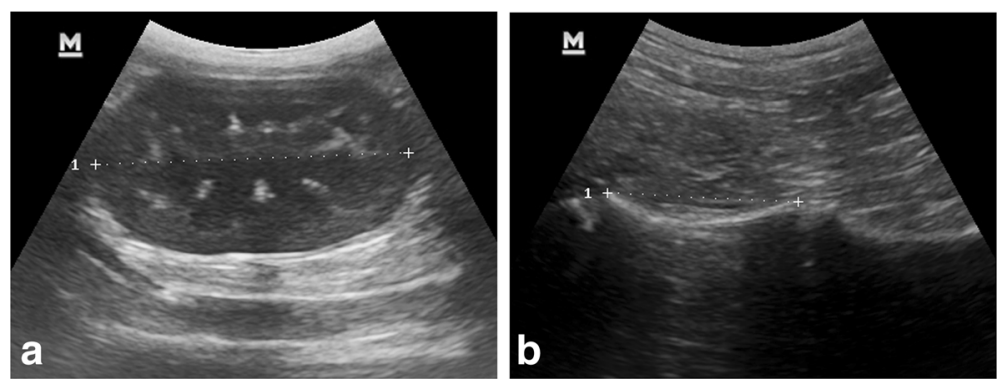

Fig. 1 a Sagittal ultrasonographic image of a normal left kidney with cursors delineating its length. b Sagittal ultrasonographic image showing the ventral border of the caudal lumbar vertebra with cursors delineating the length of L6. On both side of the cursors are the intervertebral disc spaces and to the right of the cursors the ventral border of $\mathrm{L} 7 \mathrm{can}$ be seen. Ventral is at the top of the images and cranial is to the left

between males and females and there were no significant correlations to cheetah age (Table 2).

General abdominal ultrasonography only detected incidental splenic myelolipomas in 24 cheetahs ranging in number from 1 to more than 50 in an individual cheetah and these varied in diameter from 1 to $14.9 \mathrm{~mm}$.

The kidneys appeared ultrasonographically normal and the measurements could readily be made as could the aortic luminal diameters and length of L6 (Tables 3, 4). Significant differences between male and female ultrasound measurements could only be found for aortic diameter $(P=0.006)$, right renal volume $(P=0.02)$ and right renal volume:L6 length $(P=0.03)$ with values significantly larger in males compared to females. Males had significantly longer kidneys than females but when corrected for weight this was not significant.

In comparing left and right kidney dimensions only the caudal pole height differed significantly between the two kidneys (Table 3). In calculating ultrasonographically determined ratios there were no significant differences between the two kidneys (Table 4). Interestingly, dimensions and ratios of the right kidney values were always slightly larger than those of the left kidney.

A limited number of RI values could be measured (Table 5) as anesthetic protocols had to be adapted due to several cheetahs developing post darting hyperthermia and the long recovery times of the propofol infusion

Table 2 Cheetah renal function variables (means and ranges) using Vetscan VS2

\begin{tabular}{llll}
\hline & Males & Females & $\begin{array}{l}\text { Males and } \\
\text { females }^{\text {a }}\end{array}$ \\
\hline $\begin{array}{l}\text { Number } \\
\begin{array}{l}\text { Serum creatinine } \\
(\mu \mathrm{mol} / \mathrm{L})\end{array}\end{array}$ & $240(108-463)$ & $248(179-330)$ & $243(108-463)$ \\
$\begin{array}{l}\text { Serum urea } \\
\text { (mmol/L) }\end{array}$ & $12.7(7.3-22.7)$ & $12.8(7.9-18.3)$ & $12.73(7.3-22.7)$ \\
$\begin{array}{l}\text { Urine specific } \\
\text { gravity }\end{array}$ & $1.052(1.025-1.072)$ & $1.054(1.042-1.062)$ & $1052(1025-1072)$ \\
\hline
\end{tabular}

${ }^{a}$ There were no significant differences between males and females and data were thus combined anesthetic maintenance protocol. Of the 15 kidneys evaluated 3 had an average of 2 rather than 3 measurements. The ketamine/medetomidine maintained with propofol was the only regimen with a mean value under 0.50 whilst the other 3 protocols ranged from 0.51 to 0.55 .

Significant correlations between renal measurements and serum creatinine and urea concentrations, as well as urine specific gravity values were found (Table 6). In particular left renal length correlated well with creatinine $(r=-0.66$, Fig. 2$)$ and urine specific gravity $(r=0.54$ Fig. 3) whereas left renal length:L6 correlated well with creatinine $(r=-0.6)$ and urea $(r=-0.62$, Fig. 4). Left renal length:SI correlated well with creatinine $(r=-0.63)$ and urine specific gravity $(r=0.56)$. None of the renal or other measurements correlated significantly with age.

\section{Discussion}

This was a cohort of 27 aged semi-captive cheetahs with a mean age of 9.3 years (combined male and female). There were significant differences between size and weight measurements between males and females as reported previously $[38,39]$. The serum and urine variables, as well as most of the ultrasonographic measurements, did not differ significantly between males and females and there were no correlations between any of these variables and the ages of the animals. Ultrasonographically determined lengths, widths and volumes, as well as renal length:L6 and renal length:Ao did not differ significantly between left and right kidneys but the caudal heights were significantly different $(P=0.02)$.

The mean serum creatinine concentrations for male and female cheetahs in our study (240 and $248 \mu \mathrm{mol} / \mathrm{L}$ respectively), were slightly higher than the mean values reported previously by our group in captive $(184 \mu \mathrm{mol} /$ $\mathrm{L}$, mean age $=8.5$ years) and free-ranging cheetahs $(169 \mu \mathrm{mol} / \mathrm{L}$, mean age $=4.0$ years $)[14]$, but similar to the mean concentration $(240 \mu \mathrm{mol} / \mathrm{L})$ in thirteen captive cheetahs with a mean age of 5.02 years, as reported by Holder et al. [13]. Two of the cheetahs in our study had serum creatinine concentrations above the upper limit 
Table 3 Cheetah renal measurements in $\mathrm{mm}$ or $\mathrm{mm}^{3}$

\begin{tabular}{|c|c|c|c|c|c|c|}
\hline & Measurement (mm) & $N$ & Mean & SD & Minimum & Maximum \\
\hline \multirow[t]{6}{*}{ Left kidney } & Length & 27 & 65.1 & 5.5 & 55.2 & 76.9 \\
\hline & Cranial pole width & 26 & 42.1 & 4.4 & 30.1 & 52.0 \\
\hline & Cranial pole height & 26 & 38.5 & 3.8 & 32.8 & 47.4 \\
\hline & Caudal pole width & 27 & 41.4 & 4.4 & 32.6 & 49.0 \\
\hline & Caudal pole height & 27 & $36.6^{\mathrm{a}}$ & 4.7 & 28.3 & 46.9 \\
\hline & Volume & 24 & 52.4 & 10.5 & 35.8 & 72.67 \\
\hline \multirow[t]{6}{*}{ Right kidney } & Length & 26 & 65.8 & 4.9 & 54.9 & 78.3 \\
\hline & Cranial pole width & 26 & 42.8 & 4.1 & 35.1 & 51.3 \\
\hline & Cranial pole height & 26 & 40.3 & 3.3 & 33.3 & 48.2 \\
\hline & Caudal pole width & 26 & 41.6 & 4.1 & 35.2 & 50.9 \\
\hline & Caudal pole height & 26 & $39.1^{\mathrm{a}}$ & 3.1 & 33.9 & 46.8 \\
\hline & Volume & 24 & 57.0 & 9.2 & 39.1 & 78.9 \\
\hline \multirow[t]{4}{*}{ Both kidneys ${ }^{\mathrm{b}}$} & Length & 53 & 65.4 & 5.2 & 54.9 & 78.3 \\
\hline & Cranial pole width & 52 & 42.5 & 4.2 & 30.1 & 52.0 \\
\hline & Cranial pole height & 52 & 39.4 & 3.6 & 32.8 & 48.2 \\
\hline & Caudal pole width & 53 & 41.5 & 4.2 & 32.6 & 50.9 \\
\hline
\end{tabular}

${ }^{\mathrm{a}}$ Significant difference between left and right renal measurements

${ }^{b}$ Values combined for left and right kidneys as they were not significantly different

of $300 \mu \mathrm{mol} / \mathrm{L}$ suggested by Lane et al. [15]. Interestingly these two individuals had the lowest left renal length measurements (Fig. 1), but neither showed any signs of clinical renal failure (weight loss, polyuria/polydipsia or loss of appetite). Our slightly higher creatinine values could be ascribed to the relatively old age of our cohort of cheetahs. However, the serum variables for the comparative studies were determined by different analytical methods which could also have resulted in different reference intervals. The serum urea and urine specific gravity values in our study were comparable with those previously reported $[13,14]$.

Table 4 Cheetah aorta, L6 and size index (SI) values and associated ratios

\begin{tabular}{|c|c|c|c|c|c|c|}
\hline & Measurement or ratio & $N$ & Mean & SD & Minimum & Maximum \\
\hline & Aorta diameter (mm) & 27 & 8.6 & 1.3 & 6.5 & 12.3 \\
\hline & L6 length & 27 & 40.6 & 3.1 & 33.9 & 48.8 \\
\hline \multirow[t]{5}{*}{ Left kidney } & Length:Ao & 25 & 7.69 & 1.21 & 4.54 & 10.72 \\
\hline & Volume:Ao & 24 & 6.25 & 1.39 & 2.91 & 8.55 \\
\hline & Length:L6 & 25 & 1.60 & 0.19 & 1.27 & 2.06 \\
\hline & Volume:L6 & 24 & 1.31 & 0.29 & 0.87 & 1.91 \\
\hline & Length:SI & 27 & 93.2 & 9.8 & 71.75 & 108.8 \\
\hline \multirow[t]{5}{*}{ Right kidney } & Length:Ao & 24 & 7.82 & 1.18 & 4.59 & 10.23 \\
\hline & Volume:Ao & 24 & 6.78 & 1.17 & 4.27 & 10.38 \\
\hline & Length:L6 & 24 & 1.62 & 0.17 & 1.29 & 1.96 \\
\hline & Volume:L6 & 24 & 1.42 & 0.26 & 0.94 & 1.94 \\
\hline & Length:SI & 27 & 94.0 & 8.7 & 72.65 & 107.6 \\
\hline \multirow[t]{5}{*}{ Both kidneys $^{a}$} & Length:Ao & 49 & 7.75 & 1.62 & 4.54 & 10.72 \\
\hline & Volume:Ao & 48 & 6.51 & 1.29 & 2.91 & 10.38 \\
\hline & Length:L6 & 49 & 1.61 & 0.18 & 1.27 & 1.96 \\
\hline & Volume:L6 & 48 & 1.36 & 0.28 & 0.87 & 1.94 \\
\hline & Length:SI & 54 & 93.6 & 9.1 & 71.75 & 108.8 \\
\hline
\end{tabular}


Table 5 Cheetah RI values using different immobilization and anesthetic maintenance protocols

\begin{tabular}{llllll}
\hline Immobilization & Maintenance & $N$ & Mean & SD & Range \\
\hline Ketamine/Med & Propofol & 4 & 0.46 & 0.01 & $0.44-0.47$ \\
Ketamine/Med & Isoflurane & 1 & 0.51 & - & - \\
Zoletil/Med & Propofol & 6 & 0.55 & 0.07 & $0.46-0.66$ \\
Zoletil/Med & Isoflurane & 4 & 0.54 & 0.07 & $0.45-0.61$ \\
All combined & All combined & 15 & 0.52 & 0.07 & $0.44-0.66$ \\
\hline amedetomidine & & & & &
\end{tabular}

Our renal size variables compared well to a previous ultrasonographic study of 13 younger (mean age 3.9 years) captive bred cheetahs where mean length was 63.9 (range $52-74 \mathrm{~mm}$ ) compared to our slightly longer values of 65.4 (54.9-78.3) [1]. The other size variables were also similar but were measured at different locations and could not be directly compared. Ultrasonographically determined renal length can thus be accurately measured and the technique to compare left renal length to the ultrasonographically determined L6 length would appear to be a useful dimension to detect smaller kidneys potentially associated with subclinical diffuse renal disease. The kidney length:L6 had one of the lowest relative standard deviation values of all the ratios emphasizing the value of utilizing the kidney length:L6 length as an intra cheetah evaluation of renal size irrespective of its weight.

Our study found interesting correlations between several renal measurements in relation to creatinine, urea and urine specific gravity (Table 6). Left renal length and the left renal length:SI ratios correlated most strongly with serum creatinine values $(r=0.66, P<0.0005$ in both cases), followed by the left renal length:aortic diameter $(r=0.65, P=<0.0005)$ and left renal length:L6 ratios $(r=0.60, P=0.002)$. Shorter left renal length and a lower ratios thus related to increasing creatinine and urea levels and decreasing specific gravity values. Given the high incidence of renal pathology in captive cheetahs, these ratios may well be indicative of subclinical chronic renal disease and may be useful measurements to make over time to asses renal changes in cheetahs. The results also suggest that reference intervals established for serum creatinine, urea and urine specific gravity in captive cheetahs are likely to include animals with subclinical renal disease and that serum creatinine values are perhaps more sensitive to subtle changes in renal mass than previously thought.

Furthermore, although the common renal diseases of the cheetah, glomerulosclerosis, amyloidosis and fibrosis, may be ultrasonographically detected in the advanced stage as increased cortical echogenicity as seen in dogs and cats, hyperechoic cortices may have many causes. A recent ultrasonographic - histologic study in dogs and cats determined that mean gray value (= echogenicity) was a poor test to distinguish normal from pathological kidneys with a sensitivity of $58.3 \%$ and specificity of $59.8 \%$ in dogs but with slightly better values in the domestic cat of $80.6 \%$ and $56 \%$ respectively [40]. Based on our ultrasonographic study, where no cheetahs had evidence of increased cortical echogenicity, renal size could potentially be a more important indicator of early renal pathology.

The RI values for the various darting and anesthesia maintenance protocols obtained in 15 cheetahs ranged

Table 6 Significant Pearson's correlations between renal measurements/ratios and serum creatinine, serum urea and urine specific gravity

\begin{tabular}{|c|c|c|c|c|c|c|c|}
\hline & \multirow[t]{2}{*}{$\begin{array}{l}\text { Renal } \\
\text { measurement }\end{array}$} & \multicolumn{2}{|c|}{$\begin{array}{l}\text { Serum creatinine } \\
N=27\end{array}$} & \multicolumn{2}{|c|}{$\begin{array}{l}\text { Serum urea } \\
N=27\end{array}$} & \multicolumn{2}{|c|}{$\begin{array}{l}\text { Urine SG } \\
N=24\end{array}$} \\
\hline & & $r$ & $P$ value & $r$ & $P$ value & $r$ & $P$ value \\
\hline \multirow[t]{8}{*}{ Left kidney } & Length & -0.66 & $<0.0005$ & -0.39 & 0.043 & 0.54 & 0.006 \\
\hline & Length:Size Index & -0.66 & $<0.0005$ & -0.52 & 0.006 & 0.60 & 0.002 \\
\hline & Length:Ao d & -0.65 & $<0.0005$ & -0.54 & 0.006 & 0.50 & 0.017 \\
\hline & Volume:Ao d & -0.51 & 0.011 & -0.62 & 0.001 & 0.39 & NS \\
\hline & Volume:L6 & -0.42 & 0.043 & -0.58 & 0.003 & 0.35 & NS \\
\hline & Length:L6 & -0.60 & 0.002 & -0.62 & 0.001 & 0.53 & 0.012 \\
\hline & Cranial height & -0.40 & 0.044 & -0.25 & NS & 0.43 & 0.043 \\
\hline & Volume & -0.35 & NS & -0.55 & 0.006 & 0.38 & NS \\
\hline \multirow[t]{5}{*}{ Right kidney } & Length & -0.44 & 0.023 & -0.31 & NS & 0.38 & NS \\
\hline & Length:Size Index & -0.53 & 0.006 & -0.47 & 0.015 & 0.46 & 0.025 \\
\hline & Length:Ao d & -0.52 & 0.009 & -0.48 & 0.017 & 0.40 & NS \\
\hline & Length:L6 & -0.46 & 0.025 & -0.56 & 0.004 & 0.43 & 0.045 \\
\hline & Volume:Ao d & -0.36 & NS & -0.43 & 0.034 & 0.16 & NS \\
\hline
\end{tabular}




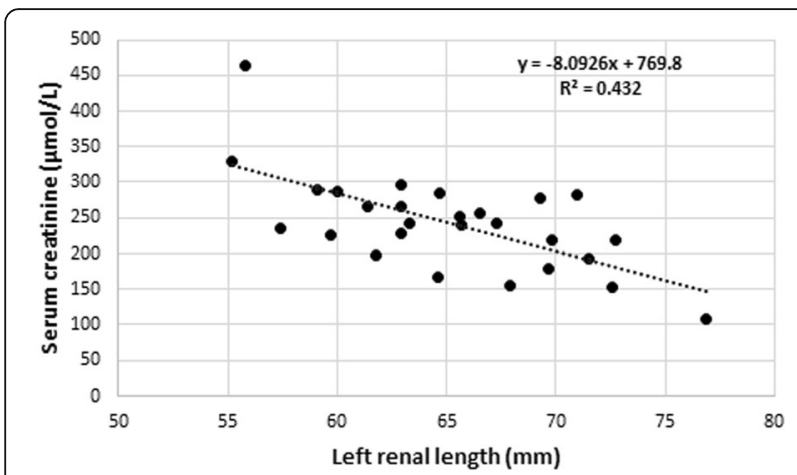

Fig. 2 Scatterplot of left renal length and serum creatinine concentrations

from 0.44 to 0.66 with a mean overall value of 0.52 . These values are comparable to those of domestic cats under different anesthetic protocols which mean values ranged from $0.52-0.90(33,34]$. These values did not differ significantly from values in awake cats [36]. Significant increased RI values have been shown in domestic cats with acute renal injury and chronic renal disease with mean values between 0.72 and 0.74 [36]. It is likely that these elevated RI values will also elevate to values $>0.65$ in some cheetah renal diseases and this could be a useful clinical field parameter to detect early renal pathology. However additional clinical data will be required to support this. Our field work will now routinely determine the RI values in at least the left kidney.

Overall left renal values appeared to have lower standard deviations than the right kidney thus making it the kidney of choice to measure ultrasonographically when evaluating kidneys for chronic renal disease likely to be affecting both kidneys. The right kidney is located more cranially and partially subcostally making ultrasonographic accessibility difficult when compared to the left kidney, which is easy to find, as the spleen may act as an acoustic window. We have reported similar findings when evaluating cheetah adrenals ultrasonographically, where the left adrenal was also easier to find and measure than

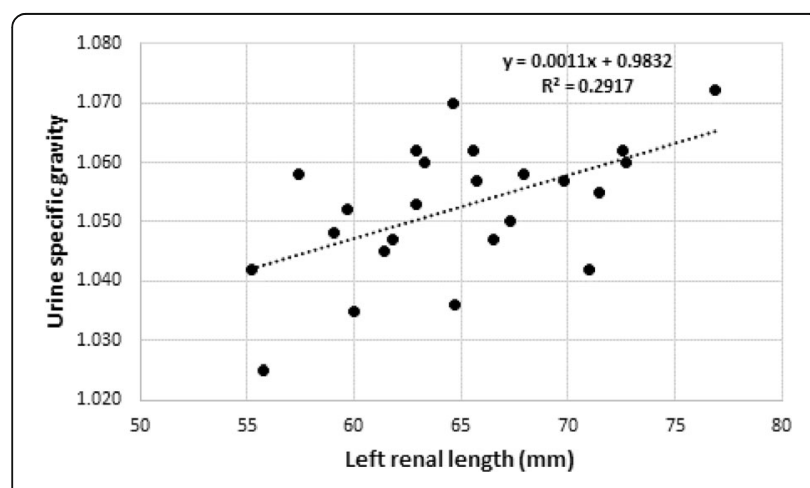

Fig. 3 Scatterplot of left renal length and urine specific gravity values

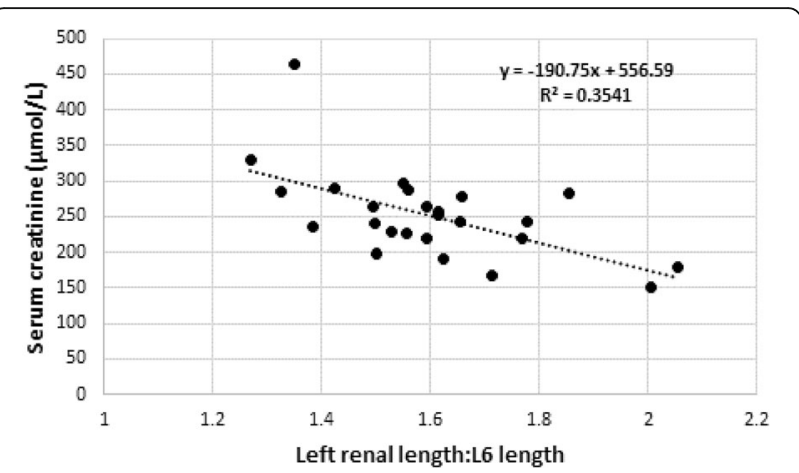

Fig. 4 Scatterplot of left renal length:L6 and serum creatinine concentrations

the right, thus resulting in more accurate adrenal measurements with lower standard deviations [2].

\section{Conclusions}

Left renal length and L6 ventral vertebral body length as well as left kidney RI values should be routinely measured in all cheetah abdominal ultrasound examinations. These values, together with serum creatinine, urea and urine specific gravity values may be relatively sensitive indicators of early renal pathology in the absence of gross ultrasonographic changes.

\section{Abbreviations}

Ao: Aorta; BL: Body length; L: Lumbar vertebra; RI: Resistive index;

SD: Standard deviation; SG: Specific gravity; SH: Shoulder height; SI: Size index

\section{Acknowledgements}

The authors extend their thanks to staff of the AfriCat Foundation in Namibia who were involved with the ultrasonographic procedures as well as handling the animals. A special thanks to the owners and directors of Africat for supporting this research project and allowing it to take place on their reserve. Thanks to Lomaen Medical for sponsoring the Mindray ultrasound machine and Dr. E H Hooijberg for evaluating the manuscript.

\section{Funding}

R Kirberger received funding from the South African National Research Foundation. The latter did not play any role in the design or conclusions of our study.

\section{Availability of data and materials}

The dataset supporting the conclusions of this article is available in the UPSpace institutional repository, http://hdl.handle.net/2263/62591

\section{Authors' contributions}

RMK designed the study, performed all the ultrasound examinations and collected the data and wrote the manuscript. ASWT performed the statistical analyses and contributed to the study design and manuscript writing. Both authors read and approved the final manuscript.

\section{Ethics approval}

The project was approved by the University's Animal Use and Care Committee and Research Committee (protocol no. V014-14) and the Research and Ethics Committee of the National Zoological Gardens of South Africa (Project no. P13/11), respectively. A research/collecting permit (no. 2013/2015) was also obtained from the Ministry of Environment and Tourism in Namibia for the project. The Africat Foundation gave written consent for the study and participated in data collection. 


\section{Consent for publication}

Not applicable

\section{Competing interests}

The authors declare that they have no competing interests.

\section{Publisher's Note}

Springer Nature remains neutral with regard to jurisdictional claims in published maps and institutional affiliations.

\section{Author details}

${ }^{1}$ Department of Companion Animal Clinical Studies, Faculty of Veterinary Science, University of Pretoria, Private Bag X04, Onderstepoort 0110, South Africa. ${ }^{2}$ Department of Paraclinical Sciences, Faculty of Veterinary Science, University of Pretoria, Private Bag X04, Onderstepoort 0110, South Africa.

${ }^{3}$ National Zoological Gardens of South Africa, Pretoria, South Africa.

Received: 27 May 2017 Accepted: 30 October 2017

Published online: 06 November 2017

\section{References}

1. Carstens A, Kirberger RM, Spotswood T, Wagner WM, Grimbeek RJ. Ultrasonography of the liver, spleen and urinary tract of the cheetah (Acinonyx jubatus). Vet Radiol Ultrasound. 2006;47:376-83.

2. Kirberger RM, Tordiffe ASW. Ultrasonographic adrenal gland findings in healthy semi-captive cheetahs (Acinonyx jubatus). Zoo Biol. 2016:35:260-8.

3. Schulman ML, Kirberger RM, Tordiffe ASW, Marker LL, Schmidt-Küntzel A, Hartman MJ. Ultrasonographic and laparoscopic evaluation of the reproductive tract in older captive female cheetahs (Acinonyx jubatus) Theriogenology. 2015;84:1611-9.

4. Munson L. Diseases of captive cheetahs (Acinonyx jubatus): results of the cheetah research council pathology survey 1989-1992. Zoo Biol. 1993;12: 105-24.

5. Munson L, Nesbit JW, Meltzer DGA, Colly LP, Bolton L, Kriek NPJ. Diseases of captive cheetahs (Acinonyx jubatus jubatus) in South Africa: a 20-year retrospective survey. J Zoo Wildl Med. 1999;30:342-7.

6. Munson L, Terrio KA, Worley M, Jago M, Bagot-Smith A, Marker L. Extrinsic factors significantly affect patterns of disease in free-ranging and captive cheetah (Acinonyx jubatus) populations. J Wildl Dis. 2005:41:542-8.

7. Papendick RE, Munson L, O'Brien TD, Johnson KH. Systemic amyloidosis in captive cheetahs (Acinonyx jubatus). Vet Pathol. 1997;34:549-56.

8. Robert N, Walzer C. Pathological disorders in captive cheetahs. In Iberian Lynx Ex Situ Conservation: An interdisciplinary approach. Vargas A, BreitenmoserWürsten C, Breitenmoser U. (Eds.). Madrid: Fundación Biodiversidad in collaboration with: IUCN Cat Specialist Group. 2009. p 265-72.

9. Gillis-Germitsch N, Vybiral PR, Codron D, Clauss M, Kotze A, Mitchell EP. Intrinsic factors, adrenal gland morphology, and disease burden in captive cheetahs (Acinonyx jubatus) in South Africa. Zoo Biol. 2016:36:40-9.

10. Url A, Krutak V, Kübber-Heiss A, Mannsberger S, Robert N, Dinhopkl N, et al. Nephropathies in the European captive cheetah (Acinonyx jubatus) population. J Zoo Wildl Med. 2016;47:797-805.

11. Hall JA, Yerramilli M, Obare E, Yerramilli M, Jewell DE. Comparison of serum concentrations of symmetric dimethylarginine and creatinine as kidney function biomarkers in cats with chronic kidney disease. J Vet Intern Med. 2014:28:1676-83

12. Nabity MB, Lees GE, Boggess MM, Yerramilli M, Obare E, Rakitin A, et al. Symmetric dimethylarginine assay validation, stability, and evaluation as a marker for the early detection of chronic kidney disease in dogs. J Vet Intern Med. 2015:29:1036-44.

13. Holder EH, Citino SB, Businga N, Cartier L, Brown SA. Measurement of glomerular filtration rate, renal plasma flow, and endogenous creatinine clearance in cheetahs (Acinonyx jubatus jubatus). J Zoo Wildl Med. 2004;35:175-8

14. Hudson-Lamb GC, Schoeman JP, Hooijberg EH, Heinrich SK, Tordiffe ASW. Reference intervals for selected serum biochemistry analytes in cheetahs (Acinonyx jubatus). J S Afr Vet Assoc. 2016;87(1):1316 6 pages. http://dx.doi. org/10.4102/2016jsava.v87i1.1316.

15. Lane EP, Miller S, Lobetti R, Caldwell P, Bertschinger HJ, Burroughs R, et al. Effect of diet on the incidence of and mortality owing to gastritis and renal disease in captive cheetahs (Acinonyx jubatus) in South Africa. Zoo Biol. 2012;31:669-82.
16. Rivers BJ, Walter PA, Polzin DJ, King VL. Duplex Doppler estimation of intrarenal Pourcelot resistive index in dogs and cats with renal disease. J Vet Intern Med. 1997;11:250-60.

17. Sanusi AA, Arogundade FA, Famurewa OC, Akintomide AO, Soyinka FO, Ojo $\mathrm{OE}$, et al. Relationship of ultrasonographically determined kidney volume with measured GFR, calculated creatinine clearance and other parameters in chronic kidney disease (CKD). Nephrol Dial Transplant. 2009;5 doi:10.1093/ndt/gfp055.

18. Nyland TG, Kantrowitz BM, Fisher P, Olander HJ, Hornof WJ. Ultrasonic determination of kidney volume in the dog. Vet Radiol Ultrasound. 1989;30: 174-80.

19. Hackendahl NC, Citino SB. Radiographic kidney measurements in captive cheetahs (Acinonyx jubatus). J Zoo Wildl Med. 2005:36:321-2.

20. Dennis R, Kirberger RM, Barr F, Wrigley RH. Handbook of small animal radiology and ultrasound. Techniques and differential diagnoses. London: Elsevier; 2010. p 117, 298-299

21. Barr FJ. Evaluation of ultrasound as a method of assessing renal size in the dog. J Small Anim Pract. 1990;31:174-9.

22. Barr FJ, Holt PE, Gibbs C. Ultrasonographic measurement of normal renal parameters. J Small Anim Pract. 1990;31:180-4.

23. Park I-C, Lee HS, Kim J-T, Nam S-J, Choi R, Oh KS, et al. Ultrasonographic evaluation of renal dimensions and resistive index in clinically healthy Korean domestic short-hair cats. J Vet Sci. 2008;9:415-9.

24. Felkai CS, Vörös K, Vrabély T, Karsai F. Ultrasonographic determination of renal volume in the dog. Vet Radiol Ultrasound. 1992;33:292-6.

25. Tyson R, Logsdon SA, Were SR, Daniel GB. Estimation of feline renal volume using computed tomography and ultrasound. Vet Radiol Ultrasound. 2013; 54:127-32.

26. Mareschal A, d'Anjou MA, Moreau M, Alexander K, Beauregard G. Ultrasonographic measurement of kidney-to-aorta ratio as a method of estimating renal size in dogs. Vet Radiol Ultrasound. 2007;83:434-8.

27. Kirberger RM. Early diagnostic imaging findings in juvenile dogs with presumed discospondylitis: 10 cases (2008-2014). J Am Vet Med Assoc. 2016;249:539-46.

28. Tublin ME, Bude RO, Platt JF. The resistive index in renal Doppler ultrasonography. Am J Res. 2003;180:885-92.

29. Cauwenberghs N, Kuznetsova T. Determinants and prognostic significance of the renal resistive index. Pulse. 2015;3:172-8.

30. Nyland TG, Fisher PE, Doverspike M, Hornhof WJ, Olander HJ. Diagnosis of urinary tract obstruction in dogs using duplex Doppler ultrasonography. Vet Radiol Ultrasound. 1993;34:348-52.

31. Rivers BJ, Walter PA, Letourneau JG, Finlay DE, Ritenour ER, King VL, et al. Duplex Doppler estimation of resistive index in arcuate arteries of sedated, normal female dogs: implications for use in the diagnosis of renal failure. J Am Anim Hosp Assoc. 1997;33:69-76.

32. Koch J, Jensen AL, Wenk A, Iversen L, Lykkegaard K. Duplex Doppler measurements of renal blood flow in a dog with Addison's disease. J Small Anim Pract. 1997:38:124-6.

33. Rivers BJ, Walter PA, O'Brien TD, Polzin DJ. Duplex Doppler estimation of Pourcelot resistive index in arcuate arteries of sedated normal cats. J Vet Intern Med. 1996;10:28-33.

34. Mitchell SK, Toal RL, Daniel GB, Rohebach BW. Evaluation of renal hemodynamics in awake and isofluorane-anesthetized cats with pulsed-wave Doppler and quantitative renal scintigraphy. Vet Radiol Ultrasound. 1998;39:451-8.

35. Carvalho CF, Chammas MC. Normal Doppler velocimetry of renal vasculature in Persian cats. J Feline Med Surg. 2011;13:399-404.

36. Tipisca V, Murino C, Cortese L, Mennonna G, Auletta L, Vulpe V, et al. Resistive index for kidney evaluation in normal and diseased cats. J Fel Med and Surg. 2016:8:471-5.

37. Mawby DI, Bartges JW, d'Avignon A, Laflamme DP, Moyers TD, Cottrell T. Comparison of various methods for estimating body fat in dogs. J Am Anim Hosp Assoc. 2004;40:109-14

38. Marker LL, Dickman AJ, Jeo RM, Mills MGL, Macdonald DW. Demography of the Namibian cheetah, Acinonyx jubatus jubatus. Biol Conserv. 2003;114:413-25.

39. Boast LK, Houser AM, Good K, Gusset M. Regional variation in body size of the cheetah (Acinonyx jubatus). J Mammal. 2013;94:1293-7.

40. Zotti A, Banzato T, Gelain ME, Centelleghe C, Vaccaro C, Aresu L. Correlation of renal histopathology with renal echogenicity in dogs and cats: an ex-vivo quantitative study. BMC Vet Res. 2015;11:99. doi:10.1186/s12917-015-0415-8. 